\title{
Corner Transition Toolpath Generation Based on Velocity-Blending Algorithm for Glass Edge Grinding
}

\author{
Kun Ren, Yujia Pan, Danyan Jiang, Jun Pan*, Wenhua Chen and Xuxiao Hu
}

\begin{abstract}
Sharp corners usually are used on glass contours to meet the highly increasing demand for personalized products, but they result in a broken wheel center toolpath in edge grinding. To ensure that the whole wheel center toolpath is of $\mathrm{G} 1$ continuity and that the grinding depth is controllable at the corners, a transition toolpath generation method based on a velocity-blending algorithm is proposed. Taking the grinding depth into consideration, the sharp-corner grinding process is planned, and a velocity-blending algorithm is introduced. With the constraints, such as traverse displacement and grinding depth, the sharp-corner transition toolpath is generated with a three-phase motion arrangement and with confirmations of the acceleration/deceleration positions. A piece of glass with three sharp corners is ground on a three-axis numerical-control glass grinding equipment. The experimental results demonstrate that the proposed algorithm can protect the sharp corners from breakage efficiently and achieve satisfactory shape accuracy. This research proposed a toolpath generation method based on a velocity-blending algorithm for the manufacturing of personalized glass products, which generates the transition toolpath as needed around a sharp corner in real time.
\end{abstract}

Keywords: Glass edge grinding, Toolpath planning, Velocity blending, Grinding depth control

\section{Introduction}

Sharp corners usually are used on glass contours to highlight a unique, personalized appearance, but they create challenges in edge grinding. In Figure 1, the center toolpath of a diamond wheel is shown with black dashed lines that are parallel to the final contour but broken at the sharp corners. Transition toolpaths should be generated for wheel traverse. Moreover, arranging a proper transition velocity profile to protect fragile tips from possible breakage should be planned carefully.

Velocity blending is one of the main techniques adopted by researchers for sharp-corner machining of metallic materials. The two data segments forming a corner are processed simultaneously by the controller to make the tool move smoothly. Accordingly, the machining efficiency is improved, but a certain contour accuracy

*Correspondence: panjun@zstu.edu.cn

College of Mechanical Engineering and Automation, Zhejiang Sci-Tech University, Hangzhou 30018, China is sacrificed. Shi et al. [1] proposed a velocity link algorithm to realize a smooth sharp-corner transition with such constraints as the corner angle, acceleration capacity, and maximum velocity limitation. Zhang et al. [2] and Tajima et al. [3] introduced the acceleration and contour error constraints to derive the velocity-blending control equations that had such parameters as motion time and transition velocity. Lee [4], Luo et al. [5], Wang [6] and Li et al. [7] adopted velocity look-ahead control strategies in kinematic planning for short linear segments to achieve maximum machining efficiency. Rewa et al. [8] proposed an asymmetrical acceleration and deceleration (acc/dec) algorithm for short-length segment machining with the acceleration limitation. With the S-type acc/ dec algorithms and limitations of acceleration and jerk, Jahanpour et al. [9], Beudaert et al. [10] and Farouki et al. [11] used Non-Uniform Rational B-Splines (NURBS) or quintic Pythagorean hodograph $(\mathrm{P}-\mathrm{H})$ curves to correct the sharp corner toolpaths and achieve velocity planning along the whole toolpath. 


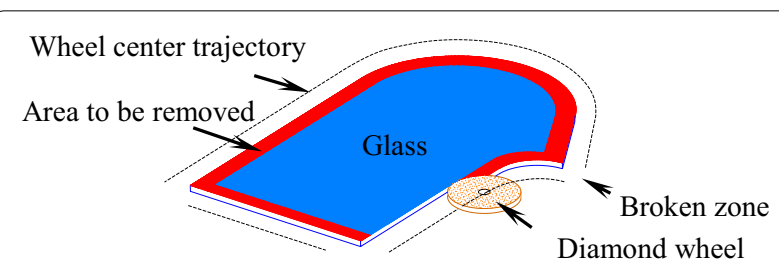

Figure 1 Glass edge grinding

The redundant material at a sharp corner is always greater than normal. Grinding force models $[12,13]$ for surface grinding of glass and other materials have been proposed. It is clear from the grinding force models that the total grinding force increases with the increase in cutting depth. Without the consideration of grinding depth control, direct application of a velocity-blending algorithm can further increase the grinding depth due to the sacrifice of contour accuracy, which results in easy corner breakage.

Corner rounding is another selection for sharp-corner machining and usually uses different kinds of parametric curves to correct the sharp corners and impose G1, G2, or higher-order continuity on the toolpaths. Zhao et al. [14] and Zhang et al. [15] used polynomial curves to correct the discrete toolpaths, which were smoothed under the constraints of maximum acceleration and velocity. Wu et al. [16], Zhao et al. [17], Pateloup et al. [18] and Lin et al. [19] pointed out the importance of toolpath planning at corners in high-speed cavity milling. Circular arcs and parametric curves were adopted to achieve tool smooth corner traverse. Duan et al. [20], Tulsyan et al. [21] and Zhou et al. [22] used NURBS curves and introduced contour error control equations to realize a shortest-time corner traverse. Ernesto et al. [23] and Bi et al. [24] used Bézier curves to interpolate the sharp corners with limitations of contour error and acceleration. Gassara et al. [25] presented a single circular arc transition method and built a velocity-planning model with a contour error constraint. Zhao et al. [26], Sencer et al. [27, 28], Beudaert et al. [29] and Pateloup et al. [30] used third- or higher-order B-spline curves to correct the sharp corners. In addition, different look-ahead control strategies with S-type acc/dec algorithms were proposed to achieve a smooth tool transition at the sharp corners.

Corner-rounding techniques can ensure that a tool traverses a sharp corner smoothly, but there is no interpolating point in the broken zones for direct curve fit, and it is time consuming for a controller to collect the proper interpolating points in real time.

To achieve sharp-corner grinding for personalized glass product manufacturing, a real-time toolpath generation method based on a velocity-blending algorithm is proposed. In Section 2, the desired sharp-corner transition

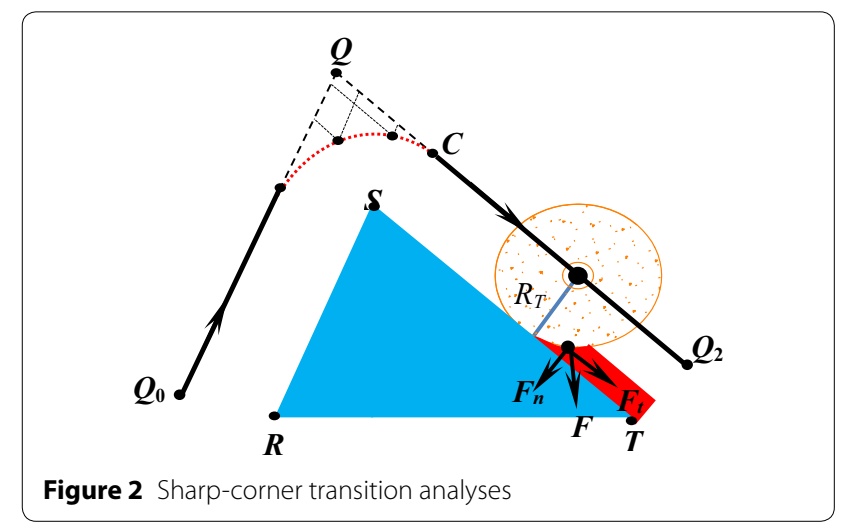

planning is proposed, and a velocity-blending algorithm is presented. Section 3 introduces the constraints, such as traverse displacement and grinding depth, to generate the transition toolpath with a three-phase motion arrangement. In Section 4, a piece of glass with three sharp corners is ground to test the performance of the proposed algorithm. Section 5 concludes the work.

\section{Toolpath-Planning Strategies}

As shown in Figure 2, a typical sharp corner is formed by two linear segments, $\overline{S R}$ and $\overline{S T} . S$ is the sharp-corner point and radius compensated to yield points $\boldsymbol{A}$ and $\boldsymbol{C}$. Obviously, the wheel center toolpath is broken between the two radius-compensated linear segments, $\overline{A Q_{0}}$ and $\overline{C Q_{2}}$ which are extended and intersect at point $Q$. The corner-grinding process starts from point $\boldsymbol{A}$ and ends at point $C$.

At the grinding zone, $\boldsymbol{F}$ is the total grinding force and composed of normal and tangential forces, $\boldsymbol{F}_{\boldsymbol{n}}$ and $\boldsymbol{F}_{\boldsymbol{t}}$. According to the grinding-force model [19], $\boldsymbol{F}$ depends on four parameters: wheel speed, feeding velocity, grinding depth, and grit diameter. Usually, wheel speed is kept constant to protect the surface from possible scratches in grinding, whereas the feeding velocity and grinding depth can be adjusted as needed.

When a wheel penetrates into the sharp-corner zone from point $\boldsymbol{A}$ (see Figure 3), the glass becomes thinner, but the redundant material becomes greater, thereby increasing the total grinding force $\boldsymbol{F}$. Especially, at point $\boldsymbol{B}$, the maximum grinding depth corresponding to the maximum $\boldsymbol{F}$ is met, and, simultaneously, the wheel moving direction has a sudden change, resulting in an easily broken corner. Therefore, when a wheel traverses a fragile tip, feeding velocity and grinding depth should be arranged properly.

In conclusion, there are three requirements for transition toolpath planning. 


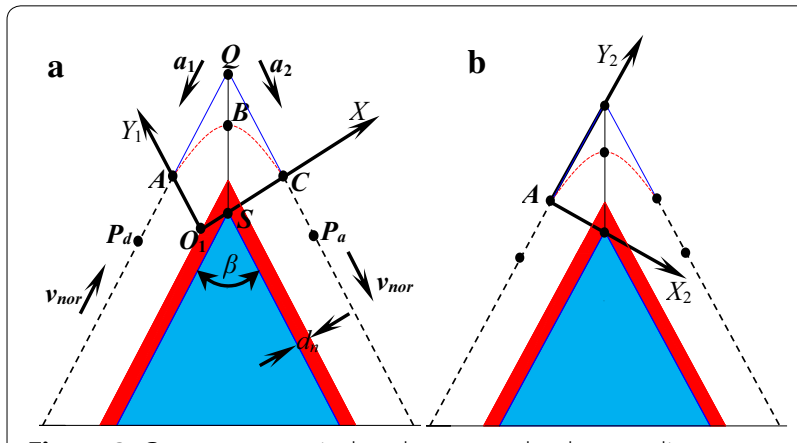

Figure 3 Corner geometrical analyses: a under the coordinate system $X_{1} O_{1} Y_{1}, \mathbf{b}$ under the coordinate system $X_{2} A Y_{2}$

(1) To limit the total grinding force $\boldsymbol{F}$, the grinding depth should be reduced gradually to be equal to or less than zero from point $\boldsymbol{A}$ to point $\boldsymbol{B}$, whereas the grinding depth should increase gradually and returns to normal from point $B$ to point $C$.

(2) To avoid a sudden change, the moving direction at points $\boldsymbol{A}$ and $\boldsymbol{C}$ should be tangent to the radiuscompensated contours, $Q_{0} A$ and $C Q_{2}$, respectively.

(3) Because of the maximum grinding depth at point $\boldsymbol{B}$, a slower transition velocity should be planned.

When the grinding wheel arrives at point $A$ with a velocity $v_{\text {trans }}$, a deceleration $\boldsymbol{a}_{1}$ is imposed on $A Q$, and, meanwhile, an acceleration $\boldsymbol{a}_{2}$ is imposed on $Q C$ (see Figure 2). With the coaction of $\boldsymbol{a}_{1}$ and $\boldsymbol{a}_{2}$, the wheel center moves to point $C$ along the transitional toolpath denoted with a red dashed line. $\boldsymbol{P}_{\boldsymbol{i}}, i \geq 0$, is an arbitrary point on the transitional toolpath and expressed as

$$
\boldsymbol{P}_{\boldsymbol{i}}=\boldsymbol{A}+\left(\boldsymbol{v}_{\text {trans }} t_{i}-\frac{1}{2} \boldsymbol{a}_{1} t_{i}^{2}\right) \boldsymbol{e}_{\boldsymbol{s}}+\frac{1}{2} \boldsymbol{a}_{2} t_{i}^{2} \boldsymbol{e}_{d}
$$

where $t_{i}$ is the elapsed blending time, $\boldsymbol{e}_{\boldsymbol{s}}$ and $\boldsymbol{e}_{\boldsymbol{d}}$ are unit vectors, and $\boldsymbol{e}_{s}=\frac{A Q}{\|A Q\|}, \boldsymbol{e}_{d}=\frac{Q C}{\|Q C\|}$. Eq. (1) gives the following.

(1) The arbitrary position $\boldsymbol{P}_{i}, i \geq 0$, is determined by $\boldsymbol{a}_{\mathbf{1}}$ and $\boldsymbol{a}_{2}$, which can be adjusted to meet requirement 1 and is planned in Section 3.

(2) The velocity is $\boldsymbol{v}_{\text {trans }} \boldsymbol{e}_{\boldsymbol{s}}$ at point $\boldsymbol{A}$ and $\boldsymbol{v}_{\text {trans }} \boldsymbol{e}_{\boldsymbol{d}}$ at point $C$, which guarantees that the generated transition toolpath is of $\mathrm{G}^{1}$ continuity and meets requirement 2 naturally.

\section{Toolpath Generation}

\subsection{Traverse Displacement Constraint}

When the wheel center arrives at point $C$, command generations for two linear segments, $\overline{A Q}$ and $\overline{Q C}$, are both finished. Along the linear segment $\overline{A Q}$,

$$
\boldsymbol{v}_{\text {trans }}=-\boldsymbol{a}_{1} t_{m},
$$

where $t_{m}$ is the total time for velocity blending from point $\boldsymbol{A}$ to point $\boldsymbol{C}$.

As shown in Figure 3(a), a local Cartesian coordinate system $X_{1} O_{1} Y_{1}$ is established. Let $O_{1}$ be the origin. In $X_{1} O_{1} Y_{1}$, the wheel center displacement $\boldsymbol{D}_{p}$ from point $\boldsymbol{A}$ to point $C$ can be derived as

$$
\boldsymbol{D}_{\boldsymbol{p}}=\left(R_{T}(1+\cos \beta),-R_{T} \sin \beta\right) .
$$

According to the kinematic theory, $\boldsymbol{D}_{\boldsymbol{p}}$ can also be derived as

$$
\boldsymbol{D}_{\boldsymbol{p}}=\left(\begin{array}{l}
\boldsymbol{v}_{\text {trans }} t_{m} \sin \beta-\frac{1}{2} \boldsymbol{a}_{1} \sin \beta t_{m}^{2}, \\
\boldsymbol{v}_{\text {trans }} t_{m} \cos \beta-\frac{1}{2}\left(\boldsymbol{a}_{1} \cos \beta+\boldsymbol{a}_{2}\right) t_{m}^{2}
\end{array}\right) .
$$

With Eqs. (3) and (4), a displacement equation can be derived as

$$
\boldsymbol{v}_{\text {trans }} t_{m} \cos \beta-\frac{1}{2}\left(\boldsymbol{a}_{1} \cos \beta+\boldsymbol{a}_{2}\right) t_{m}^{2}=-R_{T} \sin \beta .
$$

\subsection{Grinding Depth Constraint}

Connecting point $Q$ to point $S$, and when linear segment $\overline{Q S}$ is the bisector of corner angle $\beta, 0 \leq \beta \leq 180^{\circ}$. Around corner $\boldsymbol{S}$, the maximum grinding depth $d$ is

$$
d=d_{n} / \sin \left(\frac{\beta}{2}\right)
$$

where $d_{n}$ is the normal grinding depth. According to the radius compensation algorithm,

$$
\|\boldsymbol{S A}\|=\|\boldsymbol{S C}\|=R_{T},
$$

where $R_{T}$ is the wheel radius.

To simplify the calculations, as shown in Figure 3(b), a new Cartesian coordinate system $X_{2} A Y_{2}$ is established, and let point $\boldsymbol{A}$ be the origin. In $X_{2} A Y_{2}$, the corner point $\boldsymbol{S}=\left(R_{T}, 0\right)$, and an arbitrary point $\boldsymbol{P}_{\boldsymbol{i}}$ on the transition toolpath can be expressed as

$$
\boldsymbol{P}_{i}=\left(\frac{1}{2} \boldsymbol{a}_{2} t_{i}^{2} \sin \beta, \boldsymbol{v}_{\text {trans }} t_{i}-\frac{1}{2}\left(\boldsymbol{a}_{1}+\boldsymbol{a}_{2} \cos \beta\right) t_{i}^{2}\right) .
$$

Let $L(t)$ be the distance between two points, $\boldsymbol{S}$ and $\boldsymbol{P}_{i}$,

$$
\begin{aligned}
L(t)^{2}= & \left(\frac{1}{2} \boldsymbol{a}_{2} t^{2} \sin \beta-R_{T}\right)^{2} \\
& +\left(\boldsymbol{v}_{\text {trans }} t-\frac{1}{2}\left(\boldsymbol{a}_{1}+\boldsymbol{a}_{2} \cos \beta\right) t^{2}\right)^{2} .
\end{aligned}
$$

To satisfy the transition toolpath-planning requirement 1 , a grinding depth constraint is given by 


$$
L(t)^{2} \geq R_{T}^{2} .
$$

Let $F(t)=L(t)^{2}-R_{\mathcal{T}}^{2}$ and $F(t)$ is a quartic function related to time $t$. In Figure 4 , the derivatives of $F(t)$ are shown with different colors. The expressions and solutions are shown in Table 1 . Obviously, $F(t)$ is a quartic curve and has the features:

(1) If $0 \leq t \leq t_{12}, F(t)$ is increasing monotonically due to $F^{\prime}(t) \geq 0$

(2) If $t_{12}<t \leq t_{13}, F(t)$ is decreasing monotonically due to $F^{\prime}(t) \leq 0$.

Therefore, $F(t), 0 \leq t \leq t_{13}$, meets requirement 1 and can be used to design the transition toolpath. Let the total blending time $t_{m}=t_{13}$,

$$
F\left(t_{m}\right)=R_{T}^{2} \text {. }
$$

For motion symmetry, letting $t_{12}=0.5 t_{m}$, the maximum extreme value $F\left(t_{12}\right)$ should be limited by

$$
F\left(t_{12}\right) \geq\left(R_{T}+d\right)^{2} .
$$

With Eqs. (2) and (7), constraint Eq. (12) is updated by

$$
\frac{d_{n}}{R_{T}}<\left(\sqrt{1+\frac{\sin ^{2} \beta \cos \beta}{1-\cos \beta}}-1\right) \text {. }
$$

With Eqs. (5), (7), and (11), $\boldsymbol{a}_{1}$ and $\boldsymbol{a}_{2}$ can be derived as

$$
\boldsymbol{a}_{1}=\boldsymbol{a}_{2}=\frac{\boldsymbol{v}_{\text {trans }}^{2}(1-\cos \beta)}{2 R_{T} \sin \beta} .
$$

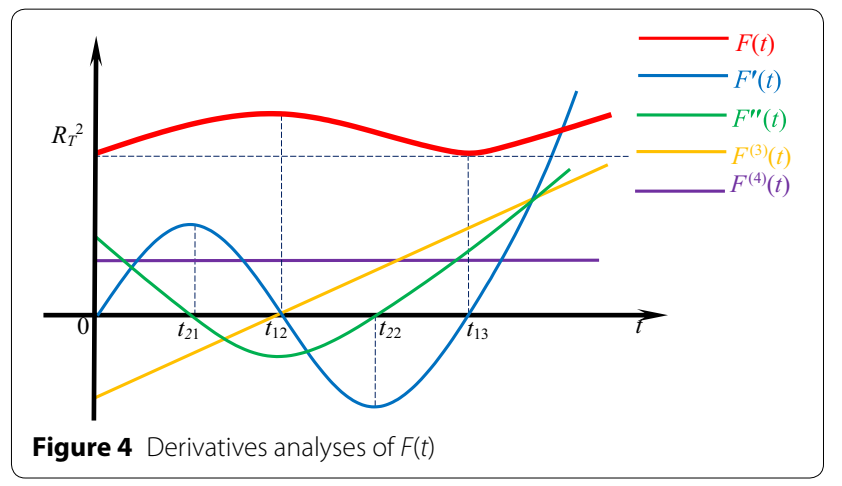

The transition toolpath is determined by $\boldsymbol{a}_{1}$ and $\boldsymbol{a}_{2}$, which can be adjusted by control Eq. (14). With the constraints in Eqs. (11) and (12), the grinding depth reduces gradually to be equal to or less than zero from point $\boldsymbol{A}$ to point $\boldsymbol{B}$, whereas the grinding depth increases gradually and returns to normal from point $B$ to point $C$. Then, the transition requirements 1 and 2 are both satisfied.

\subsection{Transition Velocity Planning}

To meet transition requirement 3 , motion through a sharp corner needs a deceleration and acceleration process. Hence, three motion phases are planned and depicted as follows:

(1) A slowing-down portion, where the velocity is decelerating from $\boldsymbol{v}_{\text {nor }}$ to $\boldsymbol{v}_{\text {trans }}$, and the deceleration distance is defined as $L_{d}$;

(2) A uniform speed portion, where tools traverse the corner from point $\boldsymbol{A}$ to point $\boldsymbol{C}$ at a constant velocity $\boldsymbol{v}_{\text {trans }}$

(3) A speed-up portion, where the velocity is accelerating from $\boldsymbol{v}_{\text {trans }}$ to $\boldsymbol{v}_{\text {nor }}$, and the acceleration distance is defined as $L_{a}$.

A linear acc/dec algorithm is utilized; thereby, the required distances for deceleration and acceleration can be figured out by

$$
L_{d}=L_{a}=\frac{\boldsymbol{v}_{\text {nor }}^{2}-\boldsymbol{v}_{\text {trans }}^{2}}{2 \sqrt{\boldsymbol{a}_{x}^{2}+\boldsymbol{a}_{y}^{2}}},
$$

where $\boldsymbol{a}_{x}$ and $\boldsymbol{a}_{y}$ are the normal accelerations of the $X$ and $Y$ axes, respectively. $\boldsymbol{v}_{\text {trans }}$ can be initialized by

$$
\boldsymbol{v}_{\text {trans }}=\frac{\beta}{180} \boldsymbol{v}_{\text {nor }} \text {. }
$$

A straight line, circular arc, and NURBS curve usually emerge before or after a corner. Ahead of the transition, a deceleration point $\boldsymbol{P}_{d}$ before point $\boldsymbol{A}$ and an acceleration point $\boldsymbol{P}_{a}$ after point $\boldsymbol{C}$ should be confirmed.

As shown in Figure 5(a), corner $S$ is formed by two linear segments that are radius compensated to yield

Table 1 Derivatives of $\boldsymbol{F}(\boldsymbol{t})$

\begin{tabular}{lll}
\hline Derivatives & Expressions & Solutions \\
\hline$F^{(4)}(t)$ & $6\left(\boldsymbol{a}_{1}^{2}+\boldsymbol{a}_{2}^{2}+2 \boldsymbol{a}_{1} \boldsymbol{a}_{2} \cos \beta\right)$ & $F^{(4)}(t)$ is a constant and greater than zero \\
$F^{(3)}(t)$ & $F^{(4)}(t) t-6 \boldsymbol{v}_{\text {trans }}\left(\boldsymbol{a}_{1}+\boldsymbol{a}_{2} \cos \beta\right)$ & $F^{(3)}(t)$ has only one solution named $t_{31}$ \\
$F^{\prime \prime}(t)$ & $-0.5 F^{(4)}(t) t^{2}+F^{(3)}(t) t+2\left(\boldsymbol{v}_{\text {trans }}^{2}-\boldsymbol{a}_{2} R \sin \beta\right)$ & $F^{\prime \prime}(t)$ has two solutions named $t_{21}$ and $t_{22}$ \\
$F^{\prime}(t)$ & $\frac{1}{3} F^{(4)}(t) t^{3}+\frac{1}{6} F^{(3)}(t) t^{2}-\frac{2}{3} F^{\prime \prime}(t) t+\frac{10}{3}\left(\boldsymbol{v}_{\text {trans }}^{2}-\boldsymbol{a}_{2} R \sin \beta\right) t$ & $F^{\prime}(t)$ has three solutions named $t_{11}, t_{12}$ and $t_{13}$ \\
\hline
\end{tabular}



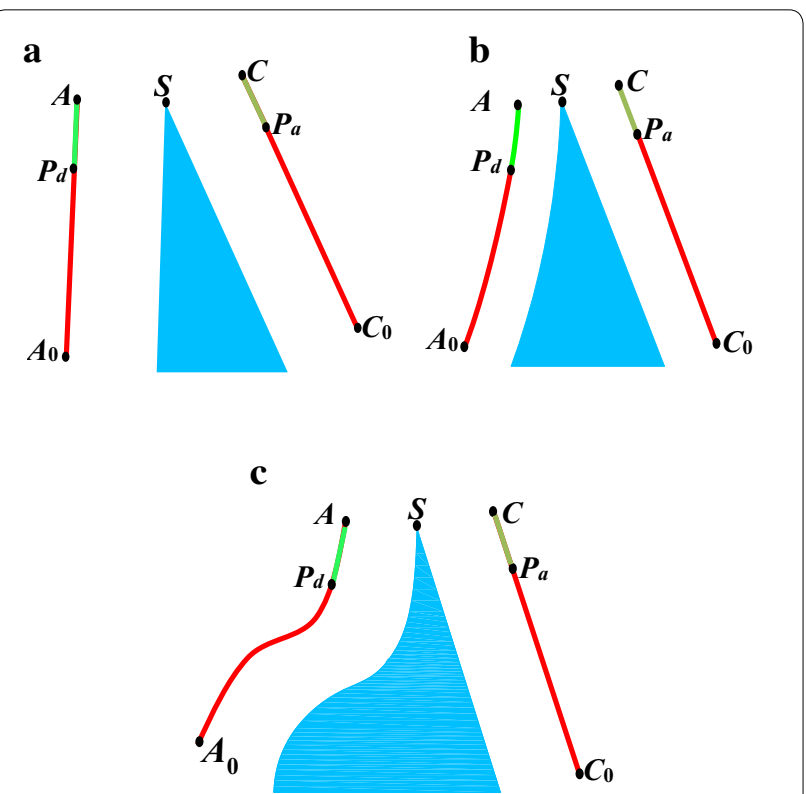

Figure $5 \mathrm{Acc} / \mathrm{dec}$ point confirmations: a for a straight line, $\mathbf{b}$ for a circular arc, $\mathbf{c}$ for a NURBS curve

$\overline{A A_{0}}$ and $\overline{C C_{0}}$. With Eq. (15), lengths $L_{d}$ and $L_{a}$ can be figured out first, so

$$
\boldsymbol{P}_{d}=\boldsymbol{A}+\frac{\boldsymbol{A}_{0}-\boldsymbol{A}}{\left\|\boldsymbol{A}_{0}-\boldsymbol{A}\right\|} L_{d}
$$

and

$$
\boldsymbol{P}_{a}=C+\frac{C_{0}-C}{\left\|C_{0}-C\right\|} L_{a} .
$$

As shown in Figure 5(b), the corner $S$ is formed by a circular arc and a straight line that are both radius compensated to yield $\widehat{A A_{0}}$ and $\overline{C C_{0}}$. Supposing that the arc center is $\boldsymbol{P}_{c}$,

$$
\boldsymbol{P}_{d}=\boldsymbol{P}_{c}+\left[\begin{array}{cc}
\cos \theta & \sin \theta \\
-\sin \theta & \cos \theta
\end{array}\right] \frac{\left(\boldsymbol{A}-\boldsymbol{P}_{c}\right)^{\mathrm{T}}}{\left\|\boldsymbol{A}-\boldsymbol{P}_{c}\right\|}
$$

where $\theta$ is decided by $L_{d}$. If $\widehat{A A_{0}}$ is clockwise, $\theta=\frac{L_{d}}{\left\|\boldsymbol{A}-\boldsymbol{P}_{c}\right\|}$; otherwise, $\theta=-\frac{L_{d}}{\left\|\boldsymbol{A}-\boldsymbol{P}_{c}\right\|}$.

As shown in Figure 5(c), the corner is formed by a straight line and a NURBS curve, which are both radius compensated to yield $\widetilde{A A_{0}}$ and $\overline{C C_{0}}$. Suppose that curve $\widetilde{A A}_{0}$ is $\boldsymbol{c}(\boldsymbol{u}), 0 \leq u \leq 1$. Let $u_{i}$ be an arbitrary knot value and $\Delta u$ be the increasing step length. Set initial knot value $u_{0}=0$ and accumulating chord length $L_{c}=0$. Let $u_{i}=u_{i-1}+\Delta u, i>0$. A point $C_{i}$ on the NURBS curve can be generated corresponding to knot value $u_{i}$, and the chord length $L_{c}$ is refreshed iteratively by

$$
L_{c}=L_{c}+\left\|C_{i}-C_{i-1}\right\| .
$$

Once $L_{c}$ is more than or equal to $L_{d}$ or $L_{a}$, the current knot value $u_{i}$ is recorded, and relevant point $\boldsymbol{P}_{d}$ or $\boldsymbol{P}_{a}$ can be confirmed.

\subsection{Trajectory Generation Steps}

Figure 6 shows the flowchart of the proposed algorithm application of which the steps are summarized briefly as follows.

\subsubsection{Step 1}

Basic parameters, such as wheel radius $R_{T}$, accelerations $a_{x}$ and $a_{y}$, glass thickness $T_{g}$, and normal grinding depth $d_{n}$ are input in the man-machine interface before machining starts.

The pattern file from a computer-aided design system is loaded into control system and then interpreted by the man-machine interface software, which is coded with Visual $\mathrm{C}++$ 6.0. G-codes representing the contour are generated, and the sharp corners along the contour are detected and marked.

With Eq. (16), $\boldsymbol{v}_{\text {trans }}$ can be determined. Taking the known $\boldsymbol{v}_{\text {trans }}$ into Eq. (14), $\boldsymbol{a}_{1}$ and $\boldsymbol{a}_{2}$ are also determined. Eq. (15) is used to figure out both $L_{d}$ and $L_{a}$. With Eqs. (17), (18), (19), and (20), points $\boldsymbol{P}_{d}$ and $\boldsymbol{P}_{a}$ are confirmed.

\subsubsection{Step 2}

The control framework with two rotary buffers is built. Each G-code segment is downloaded into the rotary buffer 1 with corresponding auxiliary machining information downloaded into the rotary buffer 2 . The auxiliary machining information is stored in a data struct that has some member variables, such as normal velocity $v_{\text {nor }}$,

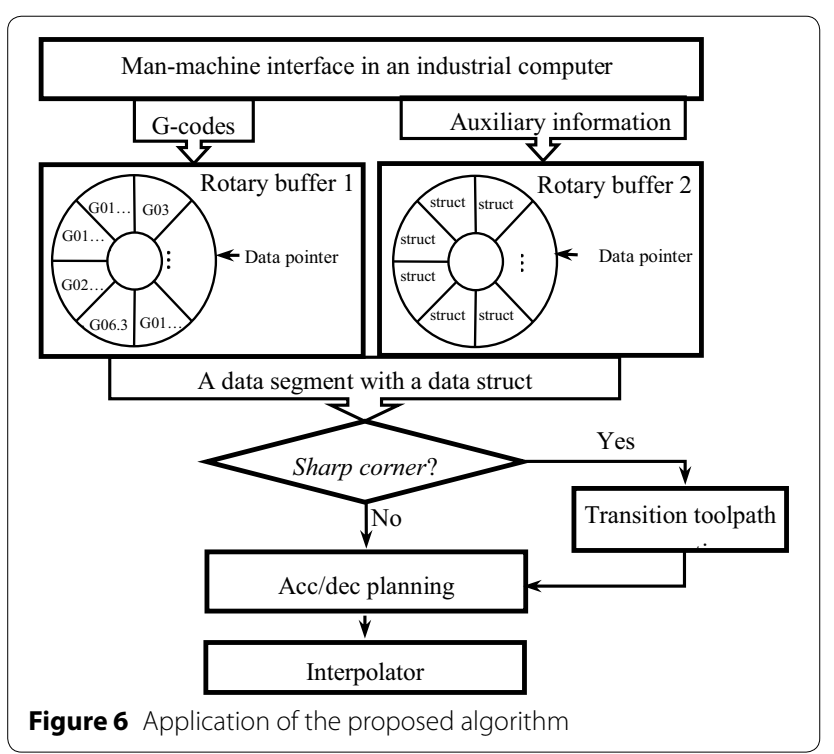


transition velocity $v_{\text {trans }}$, corner angle $\beta$, accelerations $a_{1}$ and $a_{2}$, and lengths $L_{d}$ and $L_{\mathrm{a}}$, and is defined as:

$$
\begin{aligned}
& \text { typedef struct }\{ \\
& \text { double velocitynormal; } \\
& \text { BOOL sharpcorner; } \\
& \text { BOOL datatype; } \\
& \text { double velocitytransition; } \\
& \text { double cornerangle; } \\
& \text { double acceleration; } \\
& \text { …. } \\
& \text { YINPUTPOINT; }
\end{aligned}
$$

In the INPUTPOINT struct, a Boolean variable sharpcorner is defined to mark a sharp corner, and another Boolean variable datatype is defined to mark the position of the data segment. If a sharp corner is detected, sharpcorner is true; otherwise, sharpcorner is false. If a data segment locates before the corner, datatype is false; otherwise, datatype is true.

\subsubsection{Step 3}

If sharpcorner is true, the desired transition toolpath can be generated with Eq. (1) and then inserted at the corner broken zone. After acc/dec planning is implemented, interpolation begins.

\section{Experimental Results}

The experiment was carried out with three-axis numerical-control glass grinding equipment, as shown in Figure 7 (a). The $X, Y$ and $Z$ axes traveled together to span a $1500 \times 2500 \times 120 \mathrm{~mm} 3 \mathrm{D}$ space. The experimental parameters were set as: spindle speed $S_{p}=6000 \mathrm{r} / \mathrm{min}$, normal accelerations of $X$ and $Y$ axes $a_{x}=a_{y}=20 \mathrm{~mm} / \mathrm{s}^{2}$, glass thickness $T_{g}=12 \mathrm{~mm}$, maximum grinding velocity $v_{\text {nor }}=3600 \mathrm{~mm} / \mathrm{min}$, wheel radius $R_{T}=75 \mathrm{~mm}$, normal grinding depth $d_{n}=0.13 \mathrm{~mm}$, and increasing step length $\Delta u=0.05$.

As shown in Figure 7(b), a piece of glass balustrade with three sharp corners for the escalator is being ground. Three sharp corners are numbered sequentially. Figure 8 shows the real velocity and acceleration profiles of the $X$ and $Y$ axes, respectively. The real kinematic values at the corners are shown between two vertical straight lines of different color. In addition, $v_{x}$ and $v_{y}$ denote the velocities of the $X$ and $Y$ axes, respectively.

Angle $\beta$ of corner 3 is $60^{\circ}$ and taken as an example to depict how the transition toolpath is generated and implemented. The details are as follows.

1. With Eq. (16), the transition velocity $v_{\text {trans }}=1200$ $\mathrm{mm} / \mathrm{min}$.

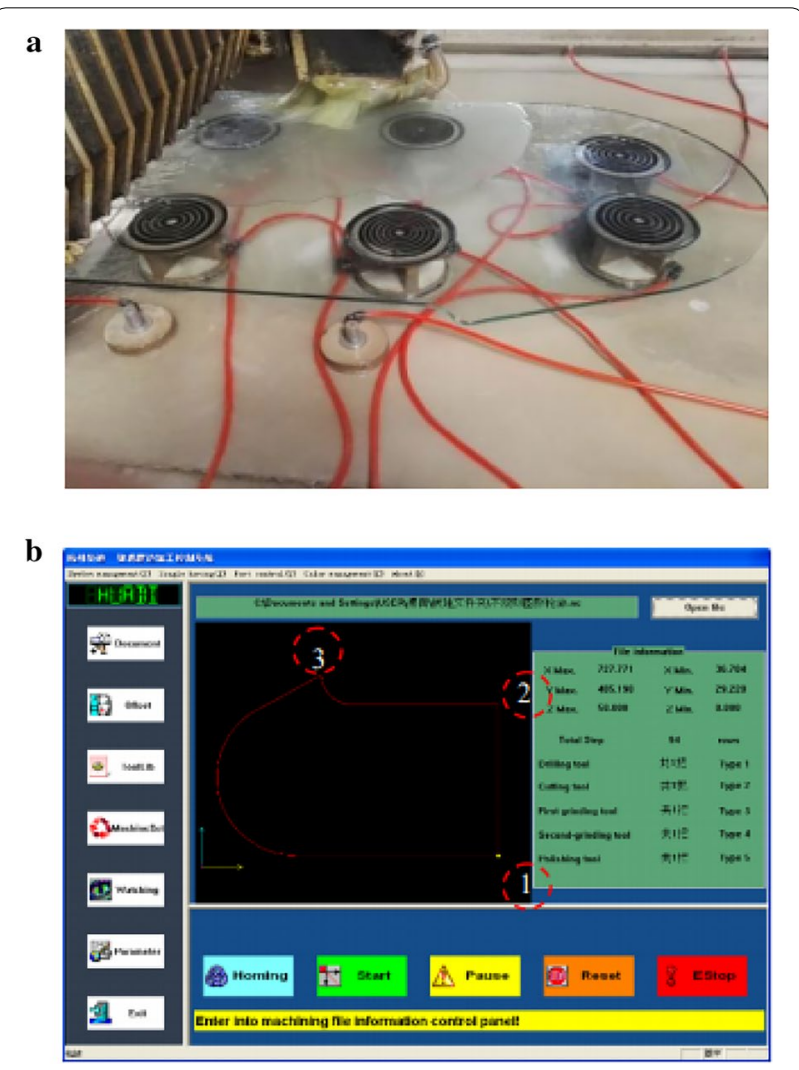

Figure 7 Experimental setup: a glass grinding center, b man-machine interface

2. Taking $\beta, v_{\text {trans }}, d_{c}$, and $R_{T}$ into Eq. (18) and checking whether the constraint is satisfied, one has $t_{12}=6 \mathrm{~s}$, and then $F\left(t_{12}\right)=8786.3 \mathrm{~mm}^{2}$ and $\left(R_{T}+\mathrm{d}\right)^{2}=5643.0$ $\mathrm{mm}^{2}$; thereby, constraint Eq. (13) is satisfied.

3. With Eq. (15), the required acc/dec lengths $L_{d}=L_{a}=56.57 \mathrm{~mm}$. Thereby,

$\boldsymbol{P}_{d}=(311.93,495.62)$,

$\boldsymbol{P}_{a}=(209.42,526.47)$.

4. With Eq. (1), the transition toolpath is generated by

$$
\boldsymbol{P}(\boldsymbol{t})=\boldsymbol{A}+\left(0.67 t^{2}, 20 t-1.15 t^{2}\right),
$$

where $0 \leq t \leq 12.9$.

The real acc/dec processes around the corners are explicitly shown in Figure 8 and in accordance with the planning. As shown in Figure 9, the wheel center trajectory is connected by the velocity-blending algorithm at each corner.

Figure 10 shows the distance change profiles between the wheel center and corner points when the diamond wheel traverses corners 1 and 3 . The position 
a
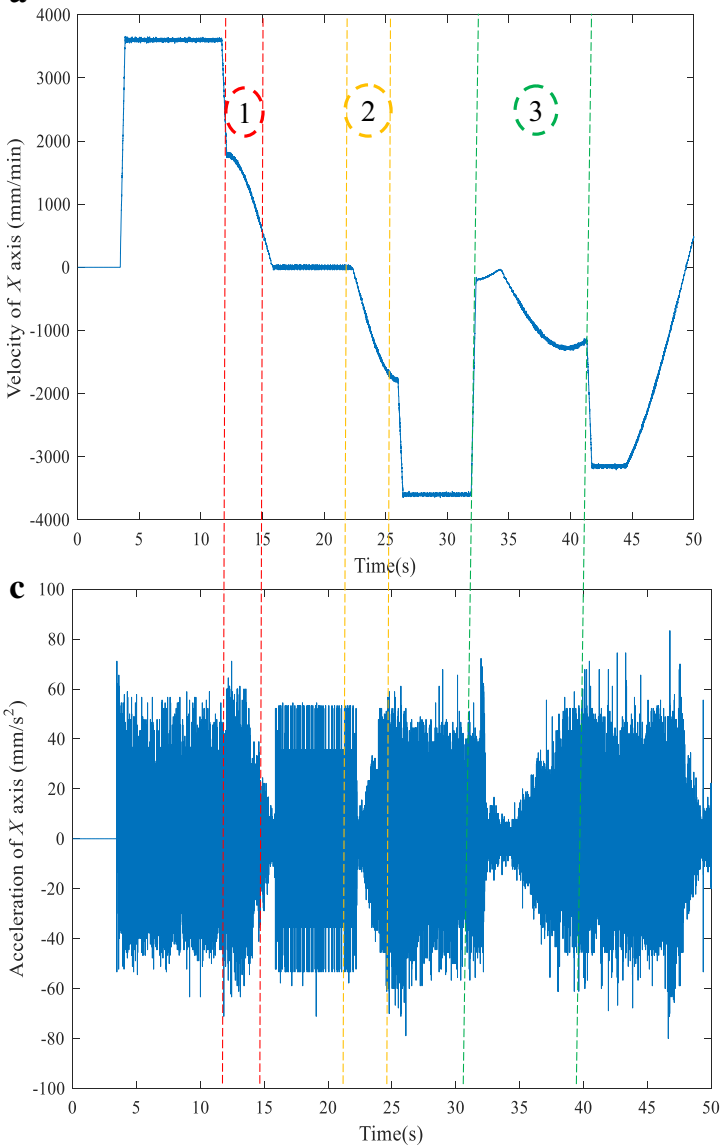

b

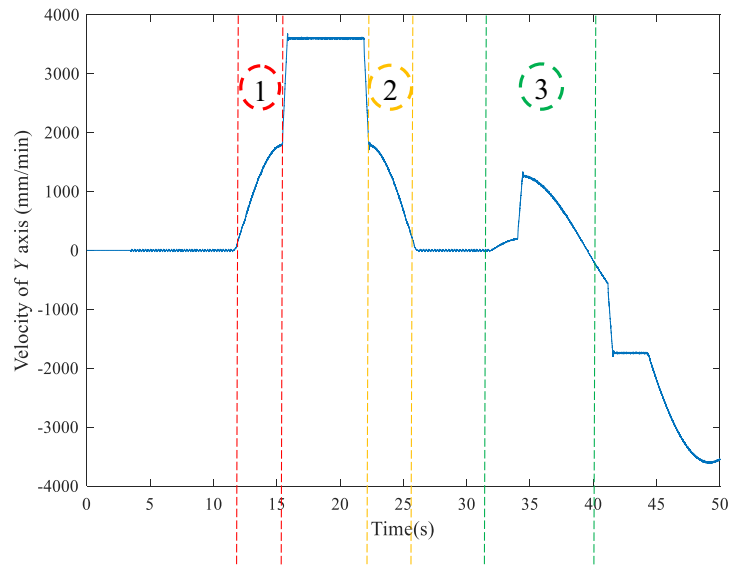

d

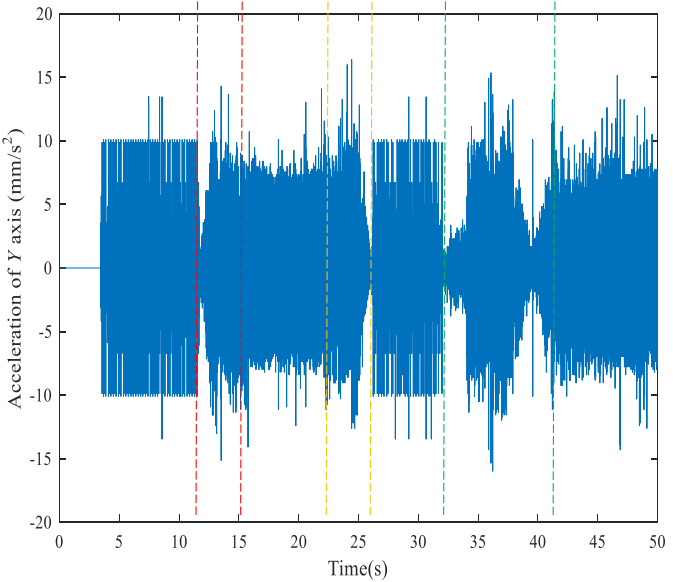

Figure 8 Kinematic profiles: a velocity profile of $X$ axis, $\mathbf{b}$ velocity profile of $Y$ axis, $\mathbf{c}$ acceleration profile of $X$ axis, $\mathbf{d}$ acceleration profile of $Y$ axis

of the $X$ axis is relative to point $A$. On the left hand of the red dashed line, the increasing distance means the gradually decreasing grinding depth; otherwise, on the right, the decreasing distance means the gradually increasing grinding depth. The distance change profiles at two different corners are both in accordance with the design requirements.

Figure 11 shows the final ground glass and its application. The proposed algorithm can protect a sharp corner from breakage efficiently, and the corner angle accuracy of $\pm 0.1^{\circ}$ fully meets the factory requirements.

\section{Conclusions}

A toolpath generation method based on a velocity-blending algorithm for the manufacturing of personalized glass products was proposed. The transition toolpath is generated based on the grinding depth and velocity control strategies, and this makes the wheel traverse a sharp corner smoothly.

Compared with the methods using velocity blending directly, the proposed algorithm uses constraints, such as traverse displacement and grinding depth, to derive an acceleration control equation that makes possible the adjustment of the transition toolpath as needed. Moreover, acc/dec distances and positions around a corner were confirmed, and they can be implemented by an interpolator easily.

Compared with other kinds of corner-rounding algorithm, the proposed algorithm generates the toolpath in real time under a control framework composed of two rotary buffers, and the main computational tasks are implemented by a powerful industrial computer, which alleviates the computational load of the control system and greatly improves the algorithm efficiency. 

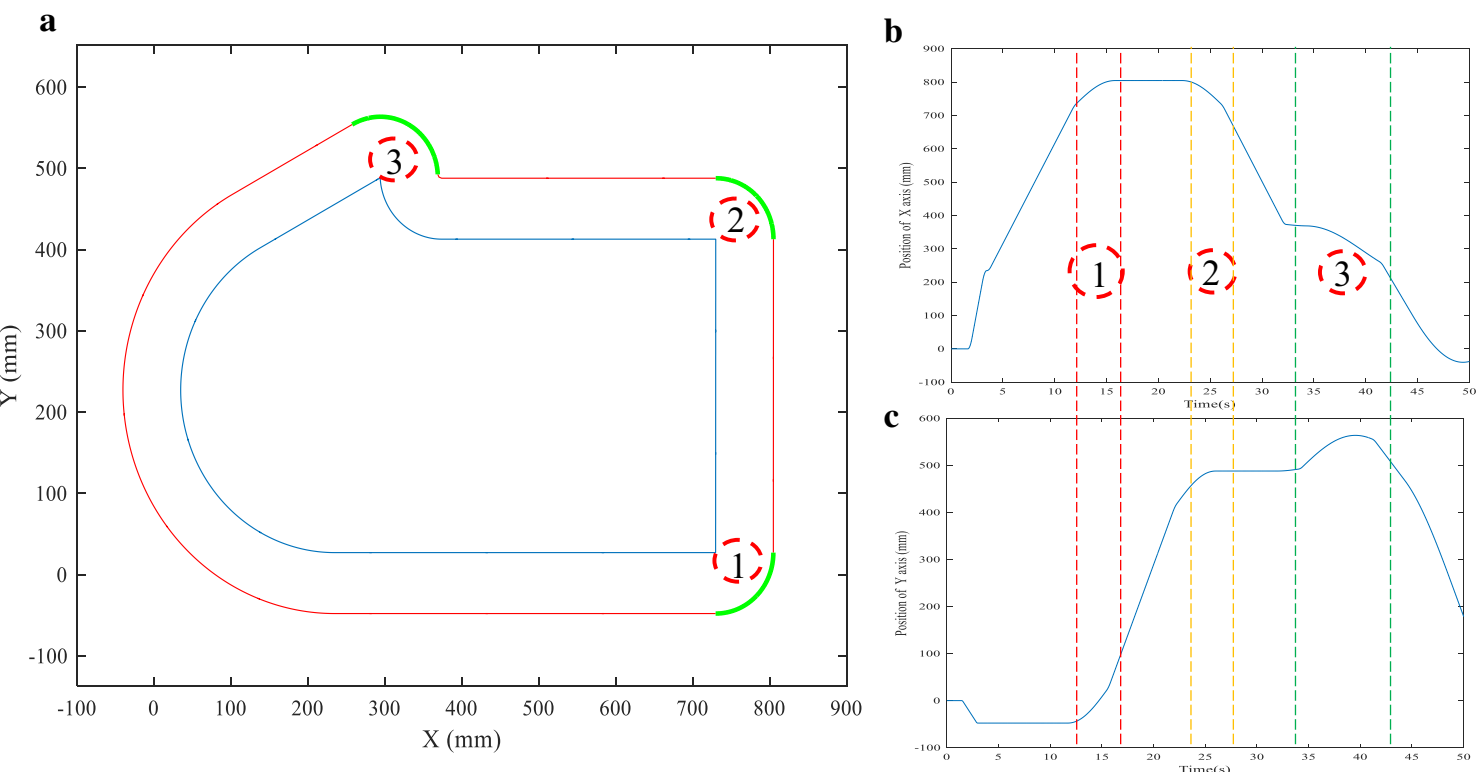

Figure 9 Generated transition toolpath at the sharp corners: a generated wheel center toolpath at the corners, $\mathbf{b}$ position profile of $X$ axis, $\mathbf{c}$ position profile of Y axis
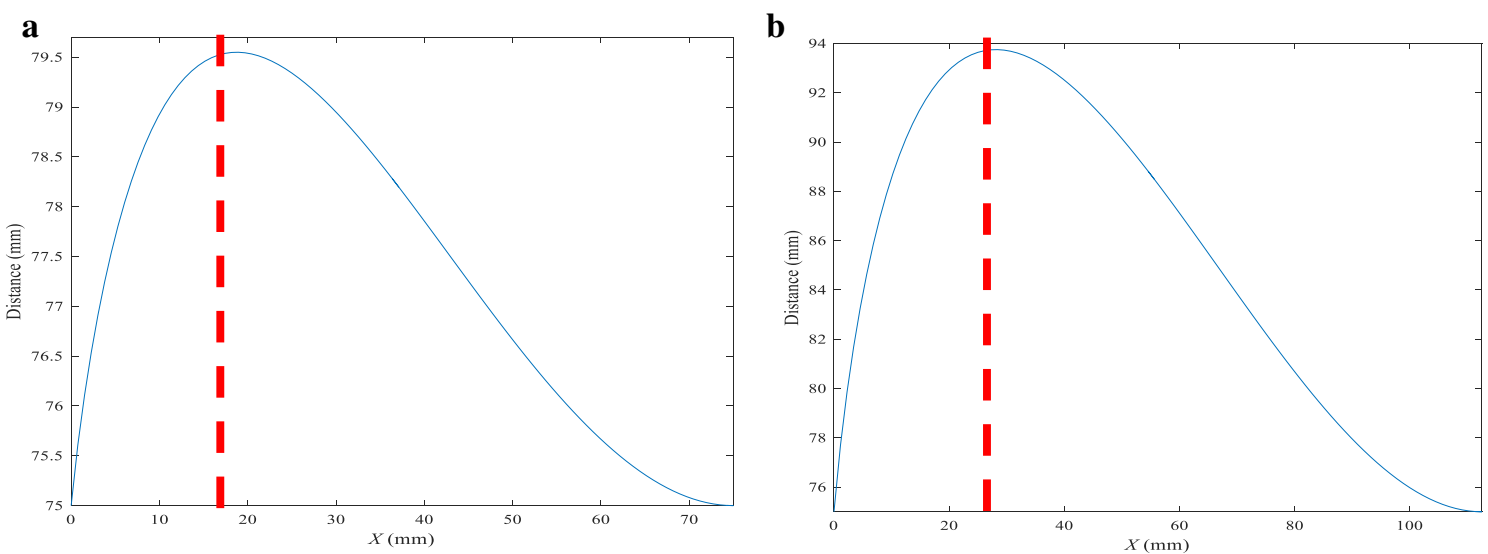

Figure 10 Distance change profiles between the wheel center and the corner points when diamond wheel traverses corners 1 and 3: a corner 1; $\mathbf{b}$ corner 3

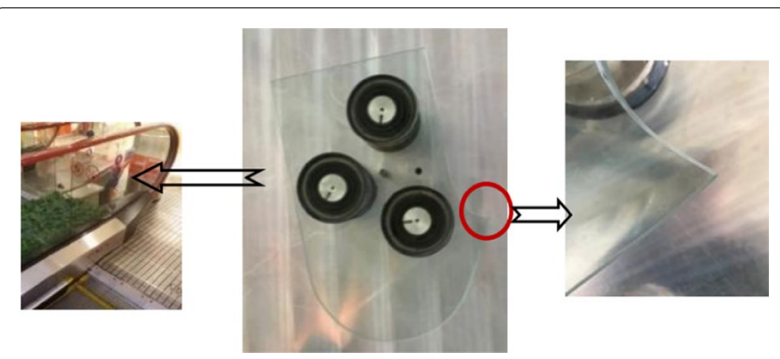

Figure 11 Grinding results
The experimental results show that the transition scheme proposed can achieve a personalized glass product with satisfactory corner shape accuracy and efficiently prevent fragile tips from breaking.

\section{Authors' Contributions}

KR carried out the toolpath-planning studies, derived the main equations and constraints, designed the experimental steps, and drafted the manuscript. YP derived some equations and collected the experimental data. DJ participated in the toolpath planning and derived some equations. JP analyzed the geometrical features of a sharp corner, proposed the transition strategies, and drafted the manuscript. WC participated in the mechanical design of the 
experimental device and helped to draft the manuscript. XH designed the control system of the experimental device. All authors read and approved the final manuscript.

\section{Authors' Information}

Kun Ren, born in 1979, is currently an associate professor at College of Mechanical Engineering and Automation, Zhejiang Sci-Tech University, China. He received his PhD degree from Zhejiang University, China, in 2008. His research interests include numerical control technology and automatic equipment development.

Yujia Pan, born in 1995, is currently a master candidate at College of Mechanical Engineering and Automation, Zhejiang Sci-Tech University, China. Danyan Jiang, born in 1993, is currently a master candidate at College of Mechanical Engineering and Automation, Zhejiang Sci-Tech University, China. Jun Pan, born in 1974, is currently a professor at College of Mechanical Engineering and Automation, Zhejiang Sci-Tech University, China.

Wenhua Chen, born in 1963, is currently a professor at College of Mechanical Engineering and Automation, Zhejiang Sci-Tech University, China.

Xuxiao Hu, born in 1965, is currently a professor at the College of Mechanical Engineering and Automation, Zhejiang Sci-Tech University, China.

\section{Competing Interests}

The authors declare that they have no competing interests.

\section{Funding}

Supported by National Key R\&D Program of China (Grant No. 2017YFB0309800), and National Natural Science Foundation of China (Grant No. 51405445)

Received: 16 January 2018 Revised: 21 July 2019 Accepted: 11 October 2019

Published online: 04 November 2019

\section{References}

[1] ZZ Shi, R F Guo, P N Li, et al. Research on transfer control strategy in CNC high-speed and high-accuracy machining. Proceedings of the 10th Annual International Conference on Computer-Aided Industrial Design and Conceptual Design, Wenzhou, China, November 26-29, 2009: 567-571.

[2] Q Zhang, X S Gao, H B Li, et al. Minimum time corner transition algorithm with confined feedrate and axial acceleration for NC machining along linear tool path. The International Journal of Advanced Manufacturing Technology, 2017, 89(1-4): 941-956.

[3] STajima, B Sencer. Kinematic corner smoothing for high speed machine tools. International Journal of Machine Tools and Manufacture, 2016, 108. 27-43.

[4] C S Lee. Generation of velocity profiles with speed limit of each axis for highspeed machining using look-ahead buffer. International Journal of Precision Engineering and Manufacturing, 2010, 11(2): 201-208.

15] FY Luo, Y F Zhou, JYin. A universal velocity profile generation approach for high-speed machining of small line segments with look-ahead. The International Journal of Advanced Manufacturing Technology, 2007, 35(5-6): 505-518.

[6] Y CWang. A look-ahead velocity blending model for high speed CNC machining of small line blocks. International Conference on Intelligent Computation Technology and Automation, Shenzhen, China, March 28-29, 2011: $555-558$.

[7] H Li, X Gao, L Zhang, et al. Discrete interpolation of G01 codes in 2D machining under bounded acceleration. Math Comput. Sci., 2012, 6(3): 327-344.

[8] K H Rewa, CW Ha, K S Kim. A practically efficient method for motion control based on asymmetric velocity profile. International Journal of Machine Tools and Manufacture, 2009, 49(7): 678-682

[9] J Jahanpour, M R Alizadeh. A novel acc-jerk-limited NURBS interpolation enhanced with an optimized S-shaped quintic feedrate scheduling scheme. The International Journal of Advanced Manufacturing Technology, 2015, 77(912): 1889-1905.
[10] X Beudaert, S Lavernhe, C Tournier. Feedrate interpolation with axis jerk constraints on 5-axis NURBS and G1 tool path. Int. J. Mach. Tools Manuf., 2012, 57(3): 73-82.

[11] RT Farouki, K M Nittler. Efficient high-speed cornering motions based on continuously-variable feedrates. I. Real-time interpolator algorithms. Int. J. Adv. Manuf. Technol., 2016, 87(9): 3557-3568.

[12] D K Fu, W F Ding, Q Miao, et al. Simulation research on the grinding forces and stresses distribution in single-grain surface grinding of Ti-6Al-4V alloy when considering the actual cutting-depth variation. Int. J. Adv. Manuf. Technol., 2017, 91(9-12): 3591-3602.

[13] U S P Durgumahanti, V Singh, PV Rao. a new model for grinding force prediction and analysis. International Journal of Machine Tools and Manufacture, 2010, 50(93): 231-240.

[14] G Y Zhao, Y G Zhao, S J Wang. The acceleration/deceleration control algorithm based on trapezoid-curve jerk in CNC machining. Research Journal of Applied Sciences, Engineering and Technology, 2013, 5(5): 1639-1645.

[15] L Zhang, Y You, J He, et al. The transition algorithm based on parametric spline curve for high-speed machining of continuous short line segments. Int. J. Adv. Manuf. Technol., 2011, 52(1): 245-254.

[16] S XWu, ZY Zhao, CYWang, et al. Optimization of toolpath with circular cycle transition for sharp corners in pocket milling. The International Journal of Advanced Manufacturing Technology, 2016, 86(9-12): 2861-2871.

[17] ZY Zhao, CY Wang, H M Zhou, et al. Pocketing toolpath optimization for sharp corners. Journal of Materials Processing Technology, 2007, 192: 175-180.

[18] V Pateloup, E Duc, P Ray. Bspline approximation of circle arc and straight line for pocket machining. Computer-Aided Design, 2010, 42(9): 817-827.

[19] Z W Lin, J Z Fu, HY Shen, et al. Smooth contour-parallel tool path generation for high-speed machining through a dual offset procedure. Int. J. Adv. Manuf. Technol., 2015, 81(5-8): 1233-1245.

[20] M Duan, C Okwudire. High speed CNC system design. Minimum-time cornering for CNC machines using an optimal control method with NURBS parameterization. The International Journal of Advanced Manufacturing Technology, 2016, 85(5-8): 1405-1418.

[21] STulsyan, Y Altintas. Local toolpath smoothing for five-axis machine tools. Int. J. Mach. Tools Manuf., 2015, 96: 15-26.

[22] B Zhou, J B Zhao, L Li, et al. NURBS curve interpolation algorithm based on tool radius compensation method. Int. J. Prod. Res., 2016, 54(15): 4448-4474.

[23] C A Ernesto, RT Farouki. High-speed cornering by CNC machines under prescribed bounds on axis accelerations and toolpath contour error. The International Journal of Advanced Manufacturing Technology, 2012, 58(1-4): 327-338.

[24] Q Z Bi, J Shi, Y HWang, et al. Analytical curvature-continuous dual-Bézier corner transition for five-axis linear tool path. Int. J. Mach. Tools Manuf., 2015, 91: 96-108.

[25] B Gassara, M Baili, G Dessein, et al. Feed rate modeling in circular-circular interpolation discontinuity for high-speed milling CNC machining of shortline segments. The International Journal of Advanced Manufacturing Technology, 2013, 65(9-12): 1619-1634.

[26] H Zhao, L M Zhu, H Ding. A real-time look-ahead interpolation methodology with curvature-continuous B-spline transition scheme for CNC machining of short line segments. International Journal of Machine Tools and Manufacture, 2013, 65(2): 88-98.

[27] B Sencer, K Ishizaki, E Shamoto. A curvature optimal sharp corner smoothing algorithm for high-speed feed motion generation of NC systems along linear tool paths. The International Journal of Advanced Manufacturing Technology, 2015, 76 (9-12): 1977-1992.

[28] B Sencer, E Shamoto. Curvature-continuous sharp corner smoothing scheme for Cartesian motion systems. 13th IEEE International Workshop on Advanced Motion Control, 2014: 374-379.

[29] X Beudaert, S Lavernhe, C Tournier. 5-axis local corner rounding of linear too path discontinuities. International Journal of Machine Tools and Manufacture, 2013, 73(10): 9-16.

[30] V Pateloup, E Duc, P Ray. B-spline approximation of circle arc and straight ine for pocket machining. Comput. Aided Des., 2010, 42(9): 817-827. 